\title{
Using the Borda rule for ranking sets of objects
}

\section{Andreas Darmann ${ }^{1} \cdot$ Christian Klamler $^{1}$}

Received: 25 November 2016 / Accepted: 16 April 2019 / Published online: 3 May 2019

(c) The Author(s) 2019

\begin{abstract}
We analyze the problem of ranking sets of objects based on a ranking over the single objects. In recent years various papers used the sum of individual scores for the objects, in particular Borda scores, to make such comparisons. The advantage of this approach lies in providing a complete ranking of sets of objects and therefore can be seen as an alternative to other methods based on best and/or worst objects. The paper contributes in two ways. On the one hand, we highlight certain drawbacks that arise when using Borda scores in such comparisons. On the other hand, we provide two characterization results for Borda-sum rankings, one for the restricted setting of sets of equal cardinality and one for the general setting which allows for comparisons of sets of unequal cardinality.
\end{abstract}

\section{Introduction}

In this work we consider the problem of ranking sets of objects based on a ranking over the single objects. Applications of such an approach do exist, for instance, in social sciences (in particular, in economics) and philosophy whenever we have to handle situations in which sets of objects need to be compared on the basis of pure ordinal information about the individual objects. This problem has been widely discussed in the social choice literature and normative justifications for such rankings over sets of objects have been provided (see Barberà et al. 2004 for an extensive survey). However, also the literature on fair division of indivisible objects is concerned with the comparison of sets of objects whenever different allocations have to be compared.

\footnotetext{
Christian Klamler

christian.klamler@uni-graz.at

Andreas Darmann

andreas.darmann@uni-graz.at

1 Institute of Public Economics, University of Graz, Graz, Austria
} 
Recently, various papers explicitly use the sum of some kind of positional scores ${ }^{1}$ with respect to the ranking of the single objects and, in particular, Borda scores ${ }^{2}$ of its respective elements (and their sum of points) to compare the different sets (see, e.g., Brams and Taylor 2000; Brams et al. 2003; Brams and King 2005; Bouveret and Lang 2011; Baumeister et al. 2017; Brams et al. 2017 or Kilgour and Vetschera 2018). In that literature the Borda-sum ranking is used to deal with issues such as efficiency, proportionality or envy-freeness, because these are hard to tackle with purely ordinal and non-additive information.

A thorough analysis of rules based on Borda scores for the objects has - to the best of our knowledge - not yet been considered. ${ }^{3}$ We fill this gap by providing axiomatic characterizations for the Borda-sum ranking. However, we will show that its use also comes at a certain price. In particular, we show that, using Borda scores, any comparison of sets of objects depends on the total number of objects available. A change in this number may change the ranking between the sets. This problem exists for all types of comparisons, whether they are between sets of equal cardinality or unequal cardinality. Nevertheless, there might still be acceptable applications for such a rule. These may, for instance, require the assumptions that the set of objects is clearly fixed and unchangeable and that we are comparing exclusively sets of equal cardinality. Given such a restricted framework, we provide a characterization of the whole family of rules based on Borda scores that compare sets of equal cardinality. In addition, we extend this characterization to rules that compare sets of unequal cardinality.

Our characterization results are, essentially, built upon four different axioms. Those contain well-known axioms from the literature, such as Extension Rule and Strict Independence, but will also introduce two new axioms. One of them, Trading, is concerned with compensating the switch in an object with its lower ranked neighbor by another switch of another object with its higher ranked neighbor. An axiom of a certain resemblance is given in Bossert and Slinko (2006) (by means of Translation Neutrality), which requires that if the best (worst) objects of two sets are translated by one position, the relative ranking of the resulting sets does not change. The second, Irrelevance of Worst Object, determines a sort of origin to the Borda score by assuming that the worst object has essentially zero value and therefore does not increase the value of a set in case it is added.

\footnotetext{
1 In general we use scoring vectors which state the amount of points assigned to the single objects with respect to their position in the ranking of the objects. For example, in a situation with $n$ objects, the famous Borda scoring vector $(n-1, n-2, \ldots, 1,0)$ assigns $n-1$ points to the top ranked object, $n-2$ points to the second ranked object, down to 0 points assigned to the bottom ranked object. Of course, many other scoring vectors are used in the literature. The evaluation of a set of objects is then determined by the sum of points assigned to its single elements.

2 Originally Borda scores have been used in determining the Borda ranking in voting situations (see, e.g., Brams and Fishburn 2002; Young 1974 and Saari 2000a,b) for such comparisons. Borda scores have, however, also been used in terms of Borda utilities in welfare economics (see, e.g., Fleurbaey and Hammond 2004).

3 An exception is a working paper by Baigent and Xu (2004) who provide a characterization of the average Borda rule.
} 
Related work. The general problem of ranking sets of objects admits many interpretations ${ }^{4}$ (Barberà et al. 2004). For instance, one could be interested in situations in which, from any set of objects, eventually one will be chosen. In that respect rankings are mostly determined by comparing either the best or worst (or both) objects in the sets. ${ }^{5}$ On the other hand, additive rules, in the more general setting of additively representable preferences over sets of $n$-tuples (via some utility representation), have also been analyzed (see, e.g., Fishburn 1970). We add to that literature by aggregating Borda scores with respect to the ranking over the single objects for the comparison of sets of objects. For the considered setting the question about additive representability of the preferences via some real-valued function $v$ is hence also related to de Finetti (1931) who provides necessary conditions for an additive representability via $v$. In a more recent work, Alcantud and Arlegi (2008) analyse the family of rankings over finite subsets of a given set that are additively representable.

This work also has a certain vicinity to the freedom of choice literature in which freedom of choice from a set of objects is evaluated based on the ranking of the objects (see, e.g., Pattanaik and Xu 1990). Our paper, however, focuses on the evaluation of sets of objects when joint use is assumed, i.e., the objects are not mutually exclusive (see, e.g., Bossert 1995). The importance of such an approach can be seen, for example, in situations where political parties negotiate over the distribution of governmental departments in a coalition, firms have to agree on a distribution of tasks among them, teams of workers have to be assigned, or sets of objects in a divorce settlement are allocated.

The paper is structured as follows: Sect. 2 introduces the formal framework. In Sect. 3 we present a paradoxical situation in connection with the Borda-sum ranking. Section 4 provides a characterization of a family of rules based on Borda scores that compare sets of equal cardinality. This is extended to a characterization of a particular family of rules for comparing sets of unequal cardinality in Sect. 5. The independence of the axioms is shown in Sect. 6 and, finally, Sect. 7 concludes the paper.

\section{Formal framework}

Let $X=\left\{x_{1}, \ldots, x_{n}\right\}$ be a non-empty, finite set of objects, and $\mathcal{X}=2^{X} \backslash \emptyset$ denote the set of all non-empty subsets of $X$. For $c \in\{1,2, \ldots, n\}$, let $\mathcal{X}_{c}$ denote the set of all size $c$ subsets of $X$. We start with a strict preference relation $P$ on $X$, where we assume $x_{1} P x_{2} P x_{3} \ldots P x_{n}$. relation $\succ$ and indifference relation $\sim$.

For any object $x_{j} \in X$, its Borda score is given by $b\left(x_{j}\right):=n-j$, for each $1 \leq$ $j \leq n$; these scores are captured by the Borda score vector $b=(n-1, n-2, \ldots, 1)$. Now, the Borda-sum ranking $\succsim_{b}$ on $\mathcal{X}$ is defined as follows: For $A, B \in \mathcal{X}$,

\footnotetext{
4 These also include decision making processes under uncertainty or ignorance, see, e.g., Gravel et al. (2018).

5 Two major results in that context are concerned with the characterization of the maximin-based ordering (e.g., Barberà et al. 1984) or the lexmin ordering (e.g., Pattanaik and Peleg 1984).
} 


$$
A \succsim_{b} B: \Longleftrightarrow \sum_{x \in A} b(x) \geq \sum_{y \in B} b(y),
$$

where $\sum_{x \in A} b(x)$ is called the score of $A$.

Clearly, any positive affine transformation $\bar{b}=\mu+\delta b$, with $\mu \geq 0$ and $\delta>0$, leads to $\succsim_{\bar{b}}$ being a weak order on $\mathcal{X}$, i.e., $\succsim_{\bar{b}}$ is a reflexive, transitive, and complete binary relation on $\mathcal{X}$. Also, note that for any positive linear transformation of Borda scores $b_{\delta}=\delta b$ with $\delta>0$, where $\succsim_{b_{\delta}}$ denotes the corresponding weak order on $X$, we have $A \succsim_{b} B \Longleftrightarrow A \succsim_{b_{\delta}} B$. That is, for any choice of $\delta>0$ using the vector $b$ or $b_{\delta}$ yields the same ranking.

In Sect. 4 we will show that, among all weak orders on $\mathcal{X}$, the order $\succsim_{b}$ (and hence the family of orders $\succsim_{\bar{b}}$; see Proposition 1 in Sect. 4), when used to compare only sets of equal cardinality, is characterized by three axioms. In Sect. 5, we will characterize the particular order $\succsim_{b}$ (and therewith the family of orders $\succsim_{b_{\delta}}$; see also Proposition 2 in Sect. 5) on the whole set $\mathcal{X}$ by the use of only one additional axiom.

For notational convenience, for any natural number $\ell<n$ let $W_{\ell}$ denote the set of the $\ell+1$ bottom ranked objects in $P$, i.e., $W_{\ell}:=\left\{x_{n-\ell}, x_{n-\ell+1}, \ldots, x_{n}\right\}$.

For any set $A \in \mathcal{X}$, let min $A$ denote the unique object $y \in A$ such that $x P y$ holds for each $x \in A \backslash\{y\}$; analogously, max $A$ denotes the unique object $z \in A$ such that $z P x$ holds for each $x \in A \backslash\{z\}$.

\section{A paradoxical situation}

In Social Choice Theory the Borda rule is used to aggregate individual preferences (rankings) into a social outcome (winning alternative(s) or a collective ranking over the alternatives) based on adding up the individual Borda scores. This is a widely discussed rule (see, e.g., Saari 2000a, b) violating, however, one of Arrow's main axioms, namely independence of irrelevant alternatives (IIA) (see Saari 1995). Intuitively, IIA implies that adding additional candidates to a voting situation should not change the social preference between any two candidates $x$ and $y$ as long as the pairwise individual preferences between $x$ and $y$ do not change. Obviously, this is not an exclusive problem of the Borda rule but comes up in any rule based on a (non-trivial) scoring vector.

Although in the current framework there is only one given preference ranking, to a certain extent, a similar problem occurs when comparing sets of objects based on Borda scores. A plausible and desirable requirement of any rule to rank sets of objects might be that the ranking of two sets does not change whenever the size of $X$, the grand set of objects, changes. In the fair division literature this might be relevant if, in a 2-agents setting, sets $A$ and $B$ are compared that do not add up to $X$. Hence, in such a situation one could think of eliminating unused objects from $X \backslash\{A \cup B\}$, but this should not change the ordering of $A$ and $B$. Of course, some division algorithms have been devised that always assign the whole set of objects to the agents, making such a requirement useless. But imagine that, in case more objects become available, any additional objects are immediately distributed among the agents as well. Then a rather weak requirement would be that if a set of objects $A$ is considered strictly better than $B$, with $A \cup B=X$, and we add two new objects $y$ and $z$ to $X$ such that $y$ is preferred 
to $z$, i.e., $y P z$, it should not be the case that now $B \cup\{z\}$ is strictly better than $A \cup\{y\}$. This can also be seen as a sort of (rather weak) monotonicity condition, typically a desirable property to be satisfied. Consider, however, the following example.

Example 1 Let $X=\left\{x_{1}, x_{2}, \ldots, x_{10}\right\}$ and the ranking $P$ be such that $x_{1} P x_{2} P \ldots P x_{10}$. The Borda score of the single objects is given by $b\left(x_{j}\right)=n-j$ for all $x_{j} \in X$, i.e., the corresponding vector of Borda scores is $b=(9,8, \ldots, 1,0)$. Let us divide $X$ into two sets $A=\left\{x_{3}, x_{4}, x_{5}, x_{6}, x_{9}\right\}$ and $B=\left\{x_{1}, x_{2}, x_{7}, x_{8}, x_{10}\right\}$. The score of $A$ is $\sum_{x \in A} b(x)=23$ and the score of $B$ is $\sum_{x \in B} b(x)=22$. Hence, $A \succ_{b} B$. Now, assume a new set of alternatives $\bar{X}=X \cup\{y, z\}$ and a new ranking $\bar{P}$, which includes $y$ and $z$, such that $x_{1} \bar{P} x_{2} \bar{P} y \bar{P} z \bar{P} x_{3} \bar{P} \ldots \bar{P} x_{10}$. Because set $\bar{X}$ has increased by two objects, the new vector of Borda scores is $(11,10,9, \ldots, 1,0)$. Let us add $y$ to the previously better set $A$ and $z$ to $B$. The new score for the set $A \cup\{y\}$ is $\sum_{x \in A \cup\{y\}} b(x)=32$ and the score for $B \cup\{z\}$ is $\sum_{x \in B \cup\{z\}} b(x)=34$. Hence, although $A$ was strictly better than $B$ when chosen from $X, A$ becomes strictly worse than $B$ under $\bar{X}$ even when we add a better object ( $y$ ) to $A$ and a worse object ( $z$ ) to $B$.

Because in the previous example we compared sets of equal cardinality the paradoxical situation occurs for any positive affine transformation of the Borda score used.

\section{Borda-sum ranking for equal cardinality}

Despite its problem discussed in the previous section, the Borda-sum ranking is actually used in various occasions and, in particular, in the literature on fair division (see, e.g., Brams and Taylor 2000; Brams et al. 2003; Brams and King 2005; Bouveret and Lang 2011; Baumeister et al. 2017; Brams et al. 2017 or Kilgour and Vetschera 2018). However, many other potential applications do exist where a comparison of sets with fixed cardinality may be relevant. These include, for example, matching theory (see, e.g., Abdulkadiroğlu and Sönmez 2013), where in many-to-one matchings certain agents (e.g., schools, universities or hospitals) compare different sets of objects (e.g., students or doctors), usually of the same cardinality, during the selection process. Moreover, in hedonic games (see, e.g., Aziz and Savani 2016), agents compare different coalitions to which they could belong to, often being of the same size, with each other. Nevertheless, in both situations it seems reasonable that there exists ordinal information about the objects contained in the set, i.e., that schools can rank the students on the market or agents can rank the other agents with whom they might potentially form a coalition.

In current approaches, comparisons between sets of objects are often incomplete or contain many indifferences if based exclusively on ordinal information about the objects. One option to overcome these problems is to use the Borda-sum of sets of objects based on the underlying Borda scores of the objects taken from the ranking. Hence, it seems useful to investigate the normative foundations of the Borda-sum ranking. In our first characterization result, we restrict ourselves to a framework in which only sets of equal cardinality are compared and all objects are allocated. 
Assume $\succsim$ to denote a weak order on $\mathcal{X}$, with asymmetric part $\succ$ and symmetric part $\sim$ respectively. Let us start with two axioms that are well-known in the literature and discussed in detail, e.g., in Barberà et al. (2004).

The first axiom, Extension Rule, provides a very intuitive idea on how to handle singleton sets: Those need to be ranked in the same way as the objects are ranked in the ranking $P$ on $X$.

Extension rule For all $x, y \in X, x P y \Longrightarrow\{x\} \succ\{y\}$.

The second axiom (Barberà and Pattanaik 1984 and see, e.g., the use of it by Foster (2011)), Strict Independence, requires the ranking of two sets of objects to be independent of the addition of any object which does not belong to those sets or the removal of an object contained in both sets. ${ }^{6}$ It can be stated as follows:

Strict independence For all $A, B \in \mathcal{X}$, for all $x \in X \backslash(A \cup B), A \succ B \Longleftrightarrow$ $A \cup\{x\} \succ B \cup\{x\}$.

The third axiom, Trading, is based on the principle of compensation, i.e., a set of objects remains essentially of identical value in case the substitution of an object by the object ranked immediately below that object in $P$ is compensated by substituting another object by the object immediately ranked above that object in $P$. This trading argument can be formalized as follows:

Trading: For all $A \in \mathcal{X}$, for all $x_{i}, x_{j} \in A$ with $x_{i+1} P x_{j-1}$ : $\left(x_{i+1} \notin A\right.$ and $\left.x_{j-1} \notin A\right) \Longrightarrow A \sim\left(A \cup\left\{x_{i+1}, x_{j-1}\right\}\right) \backslash\left\{x_{i}, x_{j}\right\}$.

As mentioned before, a possible application could be seen in the negotiation among parties about forming a coalition. This contains the distribution of ministers of governmental departments and, usually, parties have their own preference rankings over the set of departments. Because the negotiation process is not a one-shot event, during this process the negotiators of a party will have to justify the results in front of their party base. If the party is to receive only one department, Extension Rule seems very plausible. Moreover, often departments contain quite different responsibilities which are neither complementary nor substitutable, something that motivates Strict Independence. Finally, changes to a party's preliminarily assigned departments are commonly based on a compensation argument. That is, should it receive the next worse department $y$ instead of department $x$, it is compensated with the next best ministry $w$ instead of ministry $z$. This is also quite often the way how it is communicated by the negotiators to the party base. Hence, this compensation-and therefore Trading-idea is explicitly used to provide a justification for a change in the current assignment trying to make it acceptable for the stakeholders, who-as has happened in the negotiations after the German Bundestag elections 2017-may have to approve any deal of the negotiators. In that respect, it often provides a stronger argument for the negotiators to get acceptance by the stakeholders than the threat of a breakdown of the negotiations.

We will make use of the following lemma to show our first main result. The lemma states that for any weak order satisfying the axioms Extension Rule and Strict Independence, if, for any two sets $A, B$ of equal size, for each $b_{i} \in B$ and $a_{i} \in A$ we have $b_{i} P a_{i}$ or $b_{i}=a_{i}$, then $A$ cannot be preferred over $B$.

\footnotetext{
6 Barberà and Pattanaik (1984) prove that Strict Independence is incompatible with Dominance, an axiom which states that if we add to $A \subset X$ an object $x \in X \backslash A$ such that $x P y$ for all $y \in A$, then $A \cup\{x\} \succ A$ and if $y P x$ for all $y \in A$ then $A \succ A \cup\{x\}$. Dominance, however, is not satisfied by the Borda-sum ranking for any of the Borda scores, because the second part of the axiom does not hold.
} 
Lemma 1 Let $\succsim$ be a weak order on $\mathcal{X}$ that satisfies Extension Rule and Strict Independence. Let $A, B \in \mathcal{X}$ with $A=\left\{a_{1}, a_{2}, \ldots, a_{k}\right\}$ and $B=\left\{b_{1}, b_{2} \ldots, b_{k}\right\}$ such that for each $i \in\{1, \ldots, k\} b_{i}=a_{i}$ or $b_{i} P a_{i}$ holds. Then $B \succsim A$, with strict preference $B \succ A$ if $b_{i} P a_{i}$ for some $i$.

Proof Clearly, $b_{i}=a_{i}$ for each $i$ is equivalent to $B=A$, and thus $B \sim A$ follows. We distinguish the following subcases.

Case 1: $A \cap B=\emptyset$. Thus, $b_{i} P a_{i}$ for each $i \in\{1, \ldots, k\}$ follows. Extension Rule implies that

$$
\left\{b_{i}\right\} \succ\left\{a_{i}\right\}
$$

holds for each $i \in\{1, \ldots, k\}$. From (1) we know $\left\{b_{1}\right\} \succ\left\{a_{1}\right\}$. Adding $a_{2}$ to each of these sets, by Strict Independence, thus results in

$$
\left\{b_{1}, a_{2}\right\} \succ\left\{a_{1}, a_{2}\right\} .
$$

Consider the sets $\left\{a_{2}\right\}$ and $\left\{b_{2}\right\}$. We know that $\left\{b_{2}\right\} \succ\left\{a_{2}\right\}$ holds (stated in (1)); hence, by Strict Independence, adding $b_{1}$ to each of these sets yields

$$
\left\{b_{1}, b_{2}\right\} \succ\left\{b_{1}, a_{2}\right\} .
$$

With (2)

$$
\left\{b_{1}, b_{2}\right\} \succ\left\{b_{1}, a_{2}\right\} \succ\left\{a_{1}, a_{2}\right\}
$$

and due to transitivity of $\succsim$

$$
\left\{b_{1}, b_{2}\right\} \succ\left\{a_{1}, a_{2}\right\}
$$

follows.

Again due to Strict Independence, adding $a_{3}$ to each of the sets in (3) yields

$$
\left\{b_{1}, b_{2}, a_{3}\right\} \succ\left\{a_{1}, a_{2}, a_{3}\right\} .
$$

On the other hand, starting with $\left\{b_{3}\right\} \succ\left\{a_{3}\right\}$ and, in turn adding $b_{2}$ and $b_{1}$, by Strict Independence we get $\left\{b_{2}, b_{3}\right\} \succ\left\{b_{2}, a_{3}\right\}$, and, consequently, $\left\{b_{1}, b_{2}, b_{3}\right\} \succ$ $\left\{b_{1}, b_{2}, a_{3}\right\}$. With (4) and transitivity we can conclude that

$$
\left\{b_{1}, b_{2}, b_{3}\right\} \succ\left\{a_{1}, a_{2}, a_{3}\right\}
$$

holds. Now, it is not difficult to see that adding, in turn, the remaining elements of $A$ and arguing analogously for each of these elements finally yields

$$
\left\{b_{1}, b_{2}, \ldots, b_{k}\right\} \succ\left\{a_{1}, a_{2}, \ldots, a_{k}\right\}
$$


which corresponds to $B \succ A$.

Case 2: $A \cap B \neq \emptyset$. If $A \cap B \neq \emptyset$ then for $A \cap B=\left\{y_{1}, \ldots, y_{|A \cap B|}\right\}$ we have $A \succ B \Leftrightarrow A \backslash\left\{y_{1}\right\} \succ B \backslash\left\{y_{1}\right\} \Leftrightarrow A \backslash\left\{y_{1}, y_{2}\right\} \succ B \backslash\left\{y_{1}, y_{2}\right\} \Leftrightarrow \cdots \Leftrightarrow A \backslash(A \cap B) \succ$ $B \backslash(A \cap B)$ due to Strict Independence; thus, for $A^{\prime}:=A \backslash B$ and $B^{\prime}:=B \backslash A$ we have $A \succ B \Leftrightarrow A^{\prime} \succ B^{\prime}$, where $A^{\prime}$ and $B^{\prime}=\left\{b_{1}^{\prime}, \ldots, b_{\ell}^{\prime}\right\}$ are disjoint sets of equal size $\ell$ such that there are distinct elements $a_{1}^{\prime}, \ldots, a_{\ell}^{\prime} \in A^{\prime}$ with $a_{j}^{\prime} P b_{j}^{\prime}$ for each $j \in\{1, \ldots, \ell\}$, and we can repeat the above argumentation.

Now we can state the first major result of the paper, namely a characterization of the Borda-sum ranking for sets of equal cardinality. Observe that in the case of sets of equal cardinality the Borda-sum ranking is not affected by any positive affine transformation of Borda scores; i.e., for any choice of $\mu \geq 0$ and $\delta>0$ using the vector $b$ or $\bar{b}=\mu+\delta b$ will yield the same ranking. Thus, the below Theorem 1 in fact characterizes the whole family of rankings $\succsim_{\bar{b}}$.

Proposition 1 Let $c \in\{1,2, \ldots, n\}$. On $\mathcal{X}_{c}$ it holds that $\succsim_{\bar{b}}=\succsim_{b}$ for any choice of $\mu \geq 0$ and $\delta>0$.

Theorem 1 Let $c \in\{1,2, \ldots, n\}$. A weak order $\succsim$ on $\mathcal{X}_{c}$ satisfies Extension Rule, Strict Independence, and Trading if and only if $\succsim=\succsim_{b}$.

Proof It is not difficult to verify that $\succsim_{b}$ is a weak order on $\mathcal{X}_{c}$ that satisfies all of the stated axioms.

Assume the weak order $\succsim$ on $\mathcal{X}_{c}$ satisfies Extension Rule, Strict Independence, and Trading. Let $A, B \in \mathcal{X}_{c}$. In addition, let $A=\left\{x^{(1)}, x^{(2)}, \ldots, x^{(|A|)}\right\}$ where $x^{(i)} P x^{(i+1)}$ holds for any $i \in\{1, \ldots,|A|-1\}$.

We show that for any two sets $A, B \in \mathcal{X}_{c}$

$$
A \succsim B \Longleftrightarrow A \succsim_{b} B
$$

holds.

Let $|A|=|B|=c$, for some $1 \leq c \leq n$. We proceed by induction on $c$.

For $c=1$, let $A=\{x\}$ and $B=\{y\}$. If $x=y$, by reflexivity of $\succsim$ it follows that $A \sim B$ holds; clearly, $A \sim_{b} B$ holds as well. If $x \neq y$, either $x P y$ or $y P x$ is satisfied; w.l.o.g. assume $x P y$. Now, $x P y$ implies (i) $A \succ_{b} B$ by definition of $\succsim_{b}$, and (ii) $A \succ B$ due to Extension Rule. Thus, $A \succsim B \Longleftrightarrow A \succsim_{b} B$ follows.

Assume $A \succsim B \Leftrightarrow A \succsim_{b} B$ holds for all $A, B \in \mathcal{X}_{c}$ with $|A|=|B|=c$, for all $1 \leq c \leq k$, for some $k \geq 1$. Let $A, B \in \mathcal{X}_{k+1}$, i.e., with $|A|=|B|=k+1$.

Case 1: $A \cap B \neq \emptyset$. By Strict Independence, for any $x \in A \cap B$ we have $A \backslash\{x\} \succ$ $B \backslash\{x\} \Longleftrightarrow A \succ B$, and, as a consequence, $A \backslash\{x\} \sim B \backslash\{x\} \Longleftrightarrow A \sim B$. Thus,

$$
A \backslash\{x\} \succsim B \backslash\{x\} \Longleftrightarrow A \succsim B
$$

holds. Clearly, $|A \backslash\{x\}|=|B \backslash\{x\}|=k$, and by assumption

$$
A \backslash\{x\} \succsim_{b} B \backslash\{x\} \Longleftrightarrow A \backslash\{x\} \succsim B \backslash\{x\}
$$


is satisfied. With (6), we get

$$
A \backslash\{x\} \succsim_{b} B \backslash\{x\} \Longleftrightarrow A \backslash\{x\} \succsim B \backslash\{x\} \Longleftrightarrow A \succsim B
$$

Obviously, $A \backslash\{x\} \succsim_{b} B \backslash\{x\} \quad \Longleftrightarrow A \succsim_{b} B$ holds, and therefore we get $A \succsim_{b}$ $B \Longleftrightarrow A \succsim B$.

Case 2: $A \cap B=\emptyset$. Let $f: A \rightarrow B$ be the one-to-one function with $f\left(\overline{\left.x^{(i)}\right) P f}\left(x^{(i+1)}\right)\right.$ for each $i \in\{1, \ldots,|A|\}$; i.e., $f$ maps the best ranked element of $A(\max A)$ to the best ranked element of $B(\max B)$, the second best element of $A$ to the second best element of $B$, etc.

If for each $x \in A$ we have $f(x) P x$, obviously, $B \succ_{b} A$ holds; in this case, Lemma 1 yields that $B \succ A$ holds as well. Analogously, if each $x \in A$ we have $x P f(x)$, clearly $A \succ_{b} B$ follows, and due to Lemma $1 A \succ B$ is satisfied as well.

Assume there is a pair $(x, y) \in A \times A$ with $x P y$ such that (i) $f(x) P x$ and $y P f(y)$ or (ii) $x P f(x)$ and $f(y) P y$ holds. W.l.o.g. assume $x^{(1)} P f\left(x^{(1)}\right)$ (the case $f\left(x^{(1)}\right) P x^{(1)}$ can be treated in analogous manner). Let

$$
x^{(\ell)}:=\max \left\{x^{(i)} \in A \mid x^{(i)} P f\left(x^{(i)}\right) \text { and } f\left(x^{(i+1)}\right) P x^{(i+1)}\right\} .
$$

Clearly, by assumption $\ell<n$ holds and $x^{(\ell)}$ exists, i.e.,

$$
\left\{x^{(i)} \in A \mid x^{(i)} P f\left(x^{(i)}\right) \text { and } f\left(x^{(i+1)}\right) P x^{(i+1)}\right\}
$$

is non-empty. Let $x_{i}=x^{(\ell)}$ and $x_{j}=x^{(\ell+1)}$. Obviously, (7) corresponds to

$$
x_{i} P f\left(x_{i}\right) P f\left(x_{j}\right) P x_{j} .
$$

Observe that from (8) $x_{i+1} P x_{j-1}$ follows. Note that

$$
\left\{x_{i+1}, x_{i+2}, \ldots, x_{j-2}, x_{j-1}\right\} \cap A=\emptyset
$$

is implied by the definition of $x_{i}=x^{(\ell)}$ and $x_{j}=x^{(\ell+1)}$.

Due to Trading, we get $A \sim A_{1}:=\left(A \cup\left\{x_{i+1}, x_{j-1}\right\}\right) \backslash\left\{x_{i}, x_{j}\right\}$. Note that also $A \sim{ }_{b} A_{1}$ holds.

If $x_{i+1}=f\left(x_{i}\right)$ or $f\left(x_{j-1}\right)=f\left(x_{j}\right)$, then $A_{1} \cap B \neq \emptyset$, and by Case $1 A_{1} \succsim$ $B \Longleftrightarrow A_{1} \succsim_{b} B$ holds. Due to transitivity, with $A \sim A_{1}$ and $A \sim_{b} A_{1}$ we hence get $A \succsim B \Longleftrightarrow A \succsim_{b} B$.

Otherwise, by (8) there is a unique smallest number $s \in \mathbb{N}, s \geq 2$, such that $x_{i+s}=$ $f\left(x_{i}\right)$ or $x_{j-s}=f\left(x_{j}\right)$. Note that by $f\left(x_{i}\right) P f\left(x_{j}\right)$ (see (8)) we have $x_{i+h} P x_{j-h}$ for all $1 \leq h \leq s$. Let $A_{h}:=A_{h-1} \cup\left\{x_{i+h}, x_{j-h}\right\} \backslash\left\{x_{i+(h-1)}, x_{j-(h-1)}\right\}, h \in\{2, \ldots, s\}$. By repeated application of Trading we get $A_{h} \sim A_{h-1}$ for each $h \in\{2, \ldots, s\}$. By transitivity, $A_{s} \sim A_{1} \sim A$, and hence $A_{s} \sim A$ follows. Observe that $A_{s} \sim_{b} A$ holds as well. Analogously to above, $A_{s} \cap B \neq \emptyset$, and, with Case $1, A_{s} \succsim B \Longleftrightarrow A_{s} \succsim_{b} B$ follows; by transitivity, with $A \sim A_{s}$ and $A \sim_{b} A_{s}$ we can conclude that $A \succsim B \Longleftrightarrow$ $A \succsim_{b} B$ is satisfied. 


\section{Borda-sum ranking for sets of unequal cardinality}

In the previous section we provided a characterization of the Borda-sum ranking on sets of equal cardinality. In this section, we are interested in whether this can be extended to a more general characterization of the Borda-sum ranking. However, the comparison of sets of objects of unequal cardinality via the Borda-sum ranking is more demanding. Whenever we want to compare sets of unequal cardinality, there exists the issue of ambiguity, as any positive affine transformation might lead to a different ranking of the sets. ${ }^{7}$

Example 2 Let $X=\left\{x_{1}, x_{2}, x_{3}, x_{4}, x_{5}, x_{6}\right\}$ and the ranking $P$ be such that $x_{1} P x_{2} P \ldots P x_{5} P x_{6}$. Assume the two Borda score vectors $b=(5,4,3,2,1,0)$ and $\hat{b}=(6,5,4,3,2,1){ }^{8}$ As easily observed, comparing sets of equal size leads to the same ranking irrespective of the used Borda score vector. However, comparing set $A=\left\{x_{1}\right\}$ with set $B=\left\{x_{3}, x_{5}, x_{6}\right\}$ based on vector $b$, leads to $A \succ_{b} B$, as $A$ receives a score of five whereas $B$ a score of four. If we base the comparison on vector $\bar{b}$ we get $B \succ_{\bar{b}} A$, i.e., set $B$ (score of seven) is considered strictly better than set $A$ (score of six).

Hence, given the above ambiguity we will focus on one particular Borda-sum ranking, namely the one based on the most frequently used Borda score with scoring vector $b=(n-1, n-2, \ldots, 1,0)$. Actually, it can be shown that to characterize this Borda-sum ranking, we only need one additional axiom to the three axioms used before. We call this axiom Irrelevance of Worst Object. It requires that the addition of the worst object to any set not containing it does not increase its value and therefore determines an origin to our Borda scores. ${ }^{9}$ Axioms that determine origins are used in characterization results, and Irrelevance of Worst Object can, in principle, be easily enforced by adding an object of essentially zero value. However, it is worth noting that this makes the ranking vulnerable to the ambiguity issue indicated above concerning a change in the size of $X$, the total number of objects considered. Formally we state the axiom as follows:

Irrelevance of Worst Object: For all $A \in \mathcal{X}, A \sim A \cup\left\{x_{n}\right\}$.

We present two additional lemmata before providing our main result which state certain monotonicity properties.

Lemma 2 shows that for a ranking satisfying Trading, for any set $A$ there must be a set of the same cardinality that is indifferent to $A$ such that it does not contain the worst object $x_{n}$ or is contained in the set of $|A|+1$ worst objects.

Lemma 2 Let $\succsim$ be a complete binary relation on $\mathcal{X}$ that satisfies Trading. Then, for any $A \in \mathcal{X}$ with $\left|A \cap W_{|A|}\right|<|A|$ there is a set $A^{*} \in \mathcal{X}$ with $A^{*} \sim A,\left|A^{*}\right|=|A|$ such that (i) $x_{n} \notin A^{*}$ or (ii) $A^{*} \subset W_{|A|}$ holds.

\footnotetext{
7 We are grateful to an anonymous referee for highlighting this issue.

${ }^{8}$ Actually, $\hat{b}$ was the original scoring vector used by Borda, whereas $b$ is most commonly used in the literature on voting (see, e.g., Saari 1995).

${ }^{9}$ It has a certain similarity to the principle of addition of insignificant options in the literature on freedom of choice (see Jones and Sugden 1982).
} 
Proof Assume the opposite, i.e., there is a set $\tilde{A} \subset X$ with $\left|\tilde{A} \cap W_{|A|}\right|<|\tilde{A}|$ for which there is no set $A^{*} \subset X$ with $A^{*} \sim \tilde{A},\left|A^{*}\right|=|\tilde{A}|$ such that (i) $x_{n} \notin A^{*}$ or (ii) $A^{*} \subset W_{|\tilde{A}|}$ holds. Among all such sets $\tilde{A}$ let $A$ be one for which $\min (X \backslash A)$ is minimal, i.e., $\min (X \backslash \tilde{A}) P \min (X \backslash A)$ or $\min (X \backslash \tilde{A})=\min (X \backslash A)$ holds.

Clearly, $x_{n} \notin A$ contradicts with our assumption. Assume $x_{n} \in A$. Note that $\left|A \cap W_{|A|}\right|<|A|$ implies $\left|W_{|A|} \backslash A\right| \geq 2$, which, in turn, yields $|X \backslash A| \geq 2$. Let

$$
X \backslash A=\left\{y_{i_{1}}, y_{i_{2}}, \ldots, y_{i_{n-|A|}}\right\}
$$

with $y_{i_{j}} P y_{i_{j+1}}$, for each $j \in\{1, \ldots, n-|A|-1\}$. By $x_{n} \in A$, it follows that

$$
y_{i_{n-|A|}} \neq x_{n}
$$

holds. Let

$$
x_{q}:=\max \left\{x_{h} \in A \mid x_{h+1} \in X \backslash A, h \in\{1, \ldots, n-1\}\right\} .
$$

Now, $\left|A \cap W_{|A|}\right|<|A|$ implies that

- $\left\{x_{h} \in A \mid x_{h+1} \in X \backslash A, h \in\{1, \ldots, n-1\}\right\}$ is non-empty (since otherwise from (9) $A \subset W_{|A|}$ follows), and

$-x_{q+1} \neq y_{i_{n-|A|}}$ holds (and thus, in particular $x_{q+1} P y_{i_{n-|A|}}$ is satisfied), since otherwise again $A \subset W_{|A|}$ follows.

Finally, note that $y_{i_{n-|A|}} \in X \backslash A$ and $y_{i_{n-|A|}+1} \in A \backslash X$ hold. Thus, by Trading we get $A \sim A^{\prime}$, where $A^{\prime}:=A \cup\left\{x_{q+1}, y_{i_{n-|A|} \mid}\right\} \backslash\left\{x_{q}, y_{i_{n-|A|}+1}\right\}$. Clearly, $A^{\prime}$ is of the same size as $A$. Note that $\min (X \backslash A)=y_{i_{n-|A|}}$ and $\min \left(X \backslash A^{\prime}\right)=y_{i_{n-|A|}+1}$; hence, with $y_{i_{n-|A|}} P y_{i_{n-|A|}+1}$ we get $\min (X \backslash A) P \min \left(X \backslash A^{\prime}\right)$ in contradiction with the choice of A.

The following Lemma 3 states that for any weak order satisfying Extension Rule, Strict Independence and Irrelevance of Worst Object, a subset of a set $B$ cannot be preferred over $B$.

Lemma 3 Let $\succsim$ be a weak order on $\mathcal{X}$ that satisfies Extension Rule, Strict Independence and Irrelevance of Worst Object, and let $A, B \in \mathcal{X}$. Then $A \subseteq B$ implies $B \succsim A$, with strict preference $B \succ A$ if $x \in B \backslash A$ for some $x \neq x_{n}$.

Proof The proof proceeds by induction on the size $d:=|B|-|A|=|B \backslash A|$. For $d=0$ it follows that $B=A$ and hence $B \sim A$.

Assume that for some $r \geq 1$, the statement of the lemma holds for all $A, B \in \mathcal{X}$, $A \subseteq B$, with $d=r-1$. Let $A, B \in \mathcal{X}, A \subseteq B$, such that $d=r$.

Assume $x_{n} \in B \backslash A$. By Irrelevance of Worst Object $A \sim A^{\prime}:=A \cup\left\{x_{n}\right\}$ follows. Clearly, $\left|B \backslash A^{\prime}\right|=r-1$. By our induction assumption, $B \succsim A^{\prime}$ holds, with strict preference $B \succ A^{\prime}$ if $x \in B \backslash A^{\prime}$ for some $x \neq x_{n}$. With $A \sim A^{\prime}$ and transitivity of $\succsim$ the lemma follows in this case. It is not difficult to see that the case $x_{n} \in A \backslash B$ follows analogously. 
If $x_{n} \in A \cap B$, by Irrelevance of Worst Object we have $A \sim A \backslash\left\{x_{n}\right\}$ and $B \sim$ $B \backslash\left\{x_{n}\right\}$. Thus, we can restrict the attention to the remaining case of $x_{n} \notin A \cup B$. Since $x_{n} \notin A \cup B$ and $d \geq 1$ hold, there must exist $x \in B \backslash A$ with $x \neq x_{n}$. Due to Extension Rule $\{x\} \succ\left\{x_{n}\right\}$ follows. For $a \in A$ by Strict Independence it follows that $\{a, x\} \succ\left\{a, x_{n}\right\}$ holds; by repeated application of Strict Independence adding the remaining objects of $A$ yields $A \cup\{x\} \succ A \cup\left\{x_{n}\right\} \sim A$, where the indifference results from Irrelevance of Worst Object. In particular,

$$
A \cup\{x\} \succ A
$$

follows from the transitivity of $\succsim$. Observe that $B \supseteq A \cup\{x\}$ and $|B \backslash(A \cup\{x\})|=r-1$, consequently $B \succsim(A \cup\{x\})$ holds by induction assumption. With (10), $B \succsim(A \cup$ $\{x\}) \succ A$ follows which implies $B \succ A$ again due to transitivity.

Now we can state the main result of this section, namely the characterization of the Borda-sum ranking over $\mathcal{X}$ (unique up to a positive linear transformation: any vector $b_{\delta}=\delta b$ with $\delta>0$ will yield the same ranking as vector $b$ (see also Sect. 2)). Hence, Theorem 2 in fact provides a characterization for the whole family of rankings $\succsim b_{\delta}$.

Proposition 2 On $\mathcal{X}$ it holds that $\succsim_{b_{\delta}}=\succsim_{b}$ for any choice of $\delta>0$.

Theorem 2 A weak order $\succsim$ on $\mathcal{X}$ satisfies Extension Rule, Strict Independence, Irrelevance of Worst Object and Trading if and only if $\succsim_{=}{ }_{b}$.

Proof It is not difficult to verify that $\succsim_{b}$ is a weak order on $\mathcal{X}$ that satisfies all of the stated axioms.

Assume the weak order $\succsim$ on $\mathcal{X}$ satisfies Extension Rule, Strict Independence, Irrelevance of Worst Object and Trading. Let $A, B \in \mathcal{X}$, with $d:=|B|-|A|$. The goal is to show that

$$
A \succsim B \Longleftrightarrow A \succsim_{b} B
$$

holds. Let $A=\left\{x^{(1)}, x^{(2)}, \ldots, x^{(|A|)}\right\}$, where $x^{(i)} P x^{(i+1)}$ holds for any $i \in$ $\{1, \ldots,|A|-1\}$.

The proof proceeds by induction on $d$. Based on the induction assumption that (11) holds for some $d=r-1, r \geq 1$, we show that (11) is satisfied for any $A, B \in \mathcal{X}$ with $d=r$. Observe that the induction basis $(d=0)$ holds by Theorem 1 .

\section{INDUCTION ASSUMPTION}

We assume that for some $r \geq 1, A \succsim B \Longleftrightarrow A \succsim_{b} B$ holds for all $A, B \in \mathcal{X}$ with $d=r-1$.

INDUCTION STEP: $d=r$ FOR SOME $r \geq 1$.

Clearly, $|A| \neq|B|$. W.l.o.g. assume $|A|<|B|$. Consider the set

$$
W_{|A|}=\left\{x_{n-|A|}, x_{n-|A|+1}, \ldots, x_{n}\right\},
$$


i.e., the set of the $(|A|+1)$ bottom ranked objects in $P$. Note that by $|A|<|B|$ we have $A \subset X$ and thus $W_{|A|}$ is well-defined.

Case 1: $x_{n} \notin A$. Then, for the set $A^{\prime}=A \cup\left\{x_{n}\right\}$ we have $A^{\prime} \sim A$ by Irrelevance of Worst Object. Clearly, $A^{\prime} \sim_{b} A$ holds as well. Due to $|B|-\left|A^{\prime}\right|=r-1$, the induction assumption yields $A^{\prime} \succsim B \Longleftrightarrow A^{\prime} \succsim_{b} B$; by transitivity, with $A \sim A^{\prime}$ and $A \sim_{b} A^{\prime}$ we hence get $A \succsim B \Longleftrightarrow A \succsim_{b} B$.

Case 2: $x_{n} \in A$. If $x_{n} \in A$, we distinguish the following cases.

Case 2a: $A \subset W_{|A|}$. With $|B|>|A|$ and $A \subset W_{|A|}$ it follows that $B \succ_{b} A$ is satisfied. We will show that $B \succ A$ holds as well.

Let $z$ be the unique object in $W_{|A|} \backslash A$. With $x_{n} \in A, z \neq x_{n}$ follows. Thus, Lemma 3 implies $W_{|A|} \succ A$. Note that $|B|-\left|W_{|A|}\right|=r-1$. From our induction assumption we know that $B \succsim W_{|A|} \Longleftrightarrow B \succsim_{b} W_{|A|}$ holds. In fact, it is easy to see that $B \succsim_{b} W_{|A|}$ is satisfied, and therefore $B \succsim W_{|A|}$ holds. $B \succsim W_{|A|}$ and $W_{|A|} \succ A$ hence imply $B \succ A$ due to transitivity.

Case 2b: $\left|A \cap W_{|A|}\right|<|A|$. Applying Lemma 2 yields a set $A^{*}$ of the same size as $A$ such that $A^{*} \sim A$ where $x_{n} \notin A^{*}$ or $A^{*} \subset W_{|A|}$ is satisfied.

- If $x_{n} \notin A^{*}$, by Case 1 we have $A^{*} \succsim B \Longleftrightarrow A^{*} \succsim_{b} B$. It is not hard to verify that $A \sim_{b} A^{*}$ (Trading was applied to "construct" $A^{*}$ ) holds. Therewith, we can conclude $A \succsim B \Longleftrightarrow A \succsim_{b} B$ due to transitivity of $\succsim$.

- If $x_{n} \in A^{*}$, then $A^{*} \subset W_{|A|}$ holds. Thus, by Case 2a we know that both $B \succ_{b} A^{*}$ and $B \succ A^{*}$ hold, and thus $B \succ_{b} A$ and $B \succ A$ are satisfied.

Therewith, either case we have $A \succsim B \Longleftrightarrow A \succsim_{b} B$ which completes the proof.

\section{Independence of Axioms}

In this section we show the independence of the above axioms. As will be proven, whenever a weak order satisfies all but one of the above axioms, there are additional weak orders besides the Borda-sum ranking $\succsim_{b}$ that satisfy the remaining axioms.

Theorem 3 The axioms Extension Rule, Strict Independence, Irrelevance of Worst Object, and Trading are independent.

Proof In order to show independence of the axioms, we present four weak orders over $\mathcal{X}$ each of which satisfies all but one of them. Let $A, B \in \mathcal{X}$.

All but Trading. Consider the lexicographic-type ordering $\succsim_{\text {lex }}$ on $\mathcal{X}$, defined by $A \succsim_{\text {lex }} B: \Longleftrightarrow \sum_{x \in A} \ell(x) \geq \sum_{y \in B} \ell(y)$ with

$$
\ell(x)= \begin{cases}0 & \text { if } x=x_{n} \\ 2^{b(x)} & \text { otherwise }\end{cases}
$$

It is easy to verify that the weak order $\succsim_{\text {lex }}$ satisfies Extension Rule, Strict Independence and Irrelevance of Worst Object. Trading, however, is not satisfied. For instance, for any two $x_{i}, x_{j} \in A \backslash\left\{x_{n}\right\}$ with $x_{i+1} P x_{j-1}$ such that $x_{i+1} \notin A$ and $x_{j-1} \notin A$, Trading would require $\ell\left(x_{i}\right)+\ell\left(x_{j}\right)=\ell\left(x_{i+1}\right)+\ell\left(x_{j-1}\right)$ which corresponds to 
$2^{n-i}+2^{n-j}=2^{n-i-1}+2^{n-j+1}$; this, however, requires $i=j-1$ which is ruled out because $x_{i+1} P x_{j-1}$ implies $i<j-2$.

All but Irrelevance of Worst Object. Let $b^{*}\left(x_{j}\right):=b\left(x_{j}\right)+1$ for $x_{j} \in X$, i.e., $b^{*}\left(x_{j}\right)=n-j+1$. Then, it is not hard to see that the weak order $\succsim_{b^{*}}$ on $\mathcal{X}$, defined by $A \succsim_{b^{*}} B: \Longleftrightarrow \sum_{x \in A} b^{*}(x) \geq \sum_{y \in B} b^{*}(y)$ satisfies Extension Rule, Strict Independence and Trading. On the other hand, Irrelevance of Worst Object is not satisfied by $\succsim_{b^{*}}$ because of $b^{*}\left(x_{n}\right)=1$.

All but Strict Independence. Let $\succsim_{T}$ be the weak order on $\mathcal{X}$ defined as follows. For $n \neq 3$, let $\succsim_{T}$ coincide with $\succsim_{b}$. For $n=3$, let $\succsim_{T}$ be given by $\left\{x_{1}\right\} \sim_{T}\left\{x_{1}, x_{3}\right\} \succ_{T}$ $\left\{x_{1}, x_{2}\right\} \sim_{T}\left\{x_{1}, x_{2}, x_{3}\right\} \succ_{T}\left\{x_{2}\right\} \sim_{T}\left\{x_{2}, x_{3}\right\} \succ_{T}\left\{x_{3}\right\}$.

By Theorem 2, for $n \neq 3$ the weak order $\succsim_{T}$ satisfies all four axioms. Now, consider the case $n=3$. In this case, $\succsim_{T}$ satisfies Extension Rule due to $\left\{x_{1}\right\} \succ_{T}\left\{x_{2}\right\} \succ_{T}\left\{x_{3}\right\}$. In addition, $\succsim_{T}$ satisfies Irrelevance of Worst Object because of $\left\{x_{1}\right\} \sim_{T}\left\{x_{1}, x_{3}\right\}$, $\left\{x_{1}, x_{2}\right\} \sim_{T}\left\{x_{1}, x_{2}, x_{3}\right\}$, and $\left\{x_{2}\right\} \sim_{T}\left\{x_{2}, x_{3}\right\}$. Trading is trivially satisfied since $x_{i+1} P x_{j-1}$ cannot hold for any pair $\left(x_{i}, x_{j}\right)$ in the case $n=3$. On the other hand, Strict Independence is not satisfied because both $\left\{x_{2}\right\} \succ_{T}\left\{x_{3}\right\}$ and $\left\{x_{1}, x_{3}\right\} \succ_{T}\left\{x_{1}, x_{2}\right\}$ hold.

All but Extension Rule. Total indifference, i.e., the weak order $\succsim_{I}$ defined by $A \sim_{I} B$ for any $A, B \in \mathcal{X}$, obviously satisfies Strict Independence, Irrelevance of Worst Object and Trading, but does not satisfy Extension Rule.

\section{Conclusion}

In this paper we have investigated the comparison of sets of objects based on their total Borda scores of the single elements. We provided two characterizations of families of Borda-sum rankings for the comparison of sets of objects of equal and unequal cardinality. The characterization results have been achieved without explicitly using additivity assumptions. Besides two well-known axioms from the literature, Extension Rule and Strict Independence, we introduced two new (and in certain contexts reasonable) axioms, Trading and Irrelevance of Worst Object, to achieve those results. The independence of those axioms has also been established. Highlighting the drawbacks that may arise, we have shown that the use of Borda scores for such comparisons also comes at a certain price.

To some extent the paper thus provides both, arguments for and against the use of scoring vectors in the comparison of sets of objects. Hence, whether such an approach can be seen as a plausible alternative to comparisons using best and/or worst objects from the sets to be compared does still depend on the specific situation considered.

Acknowledgements Open access funding provided by University of Graz. The authors would like to thank the anonymous reviewers and the associate editor for helping to improve this paper with their valuable comments. In addition, the authors are grateful to Daniel Eckert and Walter Bossert for useful comments. This research has been supported by the University of Graz project "Voting-Selection-Decision".

Open Access This article is distributed under the terms of the Creative Commons Attribution 4.0 International License (http://creativecommons.org/licenses/by/4.0/), which permits unrestricted use, distribution, 
and reproduction in any medium, provided you give appropriate credit to the original author(s) and the source, provide a link to the Creative Commons license, and indicate if changes were made.

\section{References}

Abdulkadiroğlu A, Sönmez T (2013) Matching markets: theory and practice. In: Acemoglu D, Arellano M, Dekel E (eds) Advances in economics and econometrics: tenth world congress, vol 1. Cambridge University Press, Cambridge, pp 3-47

Alcantud JC, Arlegi R (2008) Ranking sets additively in decisional contexts: an axiomatic characterization. Theory Decis 64(2-3):147-171

Aziz H, Savani R (2016) Hedonic games. In: Brandt F, Conitzer V, Endriss U, Lang J, Procaccia AD (eds) Handbook of computational social choice, chapter 15. Cambridge University Press, Cambridge. pp $356-376$

Baigent N, Xu Y (2004) Extending an order on a set to its power set: an axiomatic characterization of the average Borda rule. Graz University: Institute of Public Economics, Mimeo

Barberà S, Pattanaik PK (1984) Extending an order on a set to the power set: some remarks on Kannai and Peleg's approach. J Econ Theory 32:185-191

Barberà S, Barrett C, Pattanaik P (1984) On some axioms for ranking sets of alternatives. J Econ Theory 33(2):301-308, 8

Barberà S, Bossert W, Pattanaik P (2004) Ranking sets of objects. In: Barberà S, Hammond P, Seidl C (eds) Handbook of utility theory: volume 2. Springer, Berlin, pp 893-977

Baumeister D, Bouveret S, Lang J, Nguyen T, Rothe J, Saffidine A (2017) Positional scoring-based allocation of indivisible goods. Auton Agents Multi-Agent Syst 31(3):628-655

Bossert W (1995) Preference extension rules for ranking sets of alternatives with a fixed cardinality. Theory Decis 39(3):301-317

Bossert W, Slinko A (2006) Relative uncertainty aversion and additively representable set rankings. Int Econ Theory 2(2):105-122

Bouveret S, Lang J (2011) A general elicitation-free protocol for allocating indivisible goods. In: IJCAI 2011. IJCAI/AAAI, pp 73-78

Brams S, King D (2005) Efficient fair division: help the worst off or avoid envy? Ration Soc 17(4):387-421

Brams S, Taylor A (2000) The win-win solution-guaranteeing fair shares to everybody. W. W. Norton \& Company, New York

Brams SJ, Fishburn PC (2002) Voting procedures. In: Arrow KJ, Sen AK, Suzumura K (eds) Handbook of social choice and welfare, volume 1, chapter 4. Elsevier, Amsterdam, pp 173-236

Brams SJ, Edelman PH, Fishburn PC (2003) Fair division of indivisible items. Theory Decis 55(2):147-180

Brams SJ, Kilgour DM, Klamler C (2017) Maximin envy-free division of indivisible items. Group Decis Negot 26(1):115-131

de Finetti B (1931) Sul significato soggettivo della probabilita. Fundam Math 17:298-329

Fishburn P (1970) Utility theory for decision making. Wiley, New York

Fleurbaey M, Hammond P (2004) Handbook of utility theory: volume 2 extensions, chapter interpersonally comparable utility. Springer, Berlin, pp 1179-1285

Foster J (2011) Freedom, opportunity, and well-being. In: Arrow K, Sen A, Suzumura K (eds) Handbook of social choice and welfare, vol 2, pp 687-728

Gravel N, Marchant T, Sen A (2018) Conditional expected utility criteria for decision making under ignorance or objective ambiguity. J Math Econ 78:79-95

Jones R, Sugden R (1982) Evaluating choice. Int Rev Law Econ 2:47-69

Kilgour DM, Vetschera R (2018) Two-player fair division of indivisible items: comparison of algorithms. Eur J Oper Res 271:620-631

Pattanaik P, Peleg B (1984) An axiomatic characterization of the lexicographic maximin extension of an ordering over a set to the power set. Soc Choice Welf 1(2):113-122

Pattanaik P, Xu Y (1990) On ranking opportunity sets in terms of freedom of choice. Rech Econ Louvain Louvain Econ Rev 56:383-390

Saari DG (1995) Basic geometry of voting. Springer, Berlin

Saari DG (2000a) Mathematical structure of voting paradoxes I: pairwise votes. Econ Theory 15(1):1-53 
Saari DG (2000b) Mathematical structure of voting paradoxes II: positional voting. Econ Theory 15(1):55102

Young H (1974) An axiomatization of Borda's rule. J Econ Theory 9(1):43-52

Publisher's Note Springer Nature remains neutral with regard to jurisdictional claims in published maps and institutional affiliations. 